\title{
A regularity result for a linear elliptic equation with Hardy-type potential
}

\author{
Ionel Ciuperca
}

Communicated by Y. Charles Li, received November 22, 2011.

\begin{abstract}
We consider a linear elliptic problem whith Dirichlet boundary conditions, with a potential term $b(x) u$ where the potential function $b$ behaves as $\frac{1}{\operatorname{dist}^{2}(x, \partial \Omega)}$ close to the boundary. We study the effect of this potential term on the $H^{2}$ regularity of the solution of the problem. An application to a stationnary Fokker-Planck-Smoluchowski equation for FENE models of diluted polymers is given.
\end{abstract}

\section{Contents}

1. Introduction 1

2. The one dimensional case 2

3. The general dimensional case 4

4. An application to a FENE model for diluted polymers 9

$\begin{array}{lr}\text { Acknowledgements } & 12\end{array}$

$\begin{array}{ll}\text { References } & 12\end{array}$

\section{Introduction}

We consider $\Omega$ an open, bounded and regular set in $\mathbb{R}^{n+1}$, with $n \in \mathrm{N}$, and $u \in H_{0}^{1}(\Omega)$ a solution of the equation

$$
-\Delta u+b(x) u=f \quad \text { in } \mathcal{D}^{\prime}(\Omega) .
$$

In the above $f$ is an element of $H^{-1}(\Omega)$ and the function $b$ is positive, regular and "behaves as $\frac{1}{\operatorname{dist}^{2}(x, \partial \Omega)}$ for $x$ close to $\partial \Omega$ ".

1991 Mathematics Subject Classification. 35H99.

Key words and phrases. Regularity of partial differential equations, Fokker-PlackSmoluchowski equations, Hardy-type potential. 
The goal of this paper is to study the influence of the term $b(x) u$ on the $H^{2}$ regularity of the solution of the problem.

Suppose for simplicity that the function $b$ is given by the expression:

$$
b(x)=\frac{b^{*}}{\operatorname{dist}^{2}(x, \partial \Omega)}
$$

where $b^{*}>0$ is a constant (more general forms of $b$ are given in Section 3 ). We prove that, under the supplementary hypothesis $b^{*}>\frac{3}{4}$, the solution $u$ belongs to $H^{2}$ for any $f \in L^{2}(\Omega)$, and we also have an appropriate inequality. Moreover, we prove that $\frac{\partial u}{\partial \nu}=0$ on $\partial \Omega$, that is, $u \in H_{0}^{2}(\Omega)$.

The $H^{2}$ regularity of $u$ in the case $b \equiv 0$ is a classical result; nevertheless, remark that the regularity result presented in this paper is not an obvious consequence of this classical regularity, since in general the term $b(x) u$ is not an element of $L^{2}(\Omega)$ under the hypothesis $u \in H_{0}^{1}(\Omega)$.

To the best of our knowledge, the $H^{2}$ regularity of the solution of such a problem was never studied in the past. For a related work, we mention the paper [1] where a problem like (1.1) is considered, with a function $b$ of the form $b(x)=\frac{b^{*}}{\|x\|^{2}}$ with $b^{*} \in \mathbb{R}$ and $0 \in \Omega$. In that paper the authors suppose that $b^{*}$ is negative with small enough absolute value and they study the $W^{1, p}$ regularity of $u$ under $L^{q}$ regularity hypothesis on $f$, with $p, q \geq 1$.

Our paper is organized as follows: In Section 2 we consider the one dimensional case $(\mathrm{n}=0)$ while in Section 3 the case $n \in \mathrm{N}^{*}$ is treated. The proof for $n \in \mathrm{N}^{*}$ is based on the result obtained in the one dimensional case. Notice that these results are stated in a more general setting than described in the beginning of this section. In Section 4 we give an application to a stationnary Fokker-Planck-Smoluchowski equation for FENE models of diluted polymers; this was the initial motivation for the result obtained in this paper.

\section{The one dimensional case}

In this section we denote $\Omega=] 0, a[$ with $a>0$ a given number. Let $f \in L^{2}(\Omega), u \in L^{2}(\Omega)$ and $b \in \mathbb{R}$ satisfying

$$
-u^{\prime \prime}(x)+\frac{b}{x^{2}} u(x)=f(x) \text { in } \mathcal{D}^{\prime}(\Omega) .
$$

Our goal is to prove a $H^{2}$ - regularity result for $u$.

The main result of this section is the following:

TheORem 2.1. Suppose that $b>\frac{3}{4}$ and let us denote

$$
\alpha \equiv \alpha(b)=\frac{1}{2}(1+\sqrt{1+4 b}) .
$$

Then for any $f \in L^{2}(\Omega)$ and $u \in L^{2}(\Omega)$ satisfying (2.1) we have:

$$
u \in H^{2}(\Omega) \quad \text { and } \quad u(0)=u^{\prime}(0)=0 .
$$

We also have the estimate

$$
\|u\|_{H^{2}(\Omega)} \leq C_{1}\|u\|_{L^{2}(\Omega)}+C_{2}\|f\|_{L^{2}(\Omega)}
$$

where

$$
C_{1}=C_{1}(a, b)=1+\frac{a}{\sqrt{2}(2 \alpha-1)}\left(\frac{\alpha}{\sqrt{2 \alpha-3}}+\frac{\alpha-1}{\sqrt{2 \alpha+1}}\right)+\frac{4 \alpha(\alpha-1)}{(2 \alpha+1)(2 \alpha-3)}+
$$




$$
+\frac{\alpha}{2(2 \alpha-1)}\left(1+\sqrt{\frac{2 \alpha+1}{2 \alpha-3}}\right)\left(\frac{\alpha-1}{\sqrt{2 \alpha-3}}+\frac{a}{\sqrt{2 \alpha-1}}\right)
$$

and

$$
C_{2}=C_{2}(a, b)=1+\frac{\alpha \sqrt{2 \alpha+1}}{a^{2}}\left(\frac{a}{\sqrt{2 \alpha-1}}+\frac{\alpha-1}{\sqrt{2 \alpha-3}}\right) .
$$

ProOF. We solve the equation (2.1) by using the change of variables

$$
\left.x=e^{t} \quad \text { with } t \in\right]-\infty, \log a[.
$$

Since

we obtain from (2.1):

$$
\frac{d^{2} u}{d x^{2}}=\frac{1}{x^{2}} \frac{d^{2} u}{d t^{2}}-\frac{1}{x^{2}} \frac{d u}{d t}
$$

$$
\frac{d^{2} u}{d t^{2}}-\frac{d u}{d t}-b u=-e^{-2 t} f
$$

We easily find that the general solutions of (2.3) is given by

$$
\begin{aligned}
u(t)= & e^{\alpha t}\left[\beta_{1}-\frac{1}{2 \alpha-1} \int_{\log (a)}^{t} e^{(2-\alpha) t^{\prime}} f\left(t^{\prime}\right) d t^{\prime}\right] \\
& +e^{(1-\alpha) t}\left[\beta_{2}+\frac{1}{2 \alpha-1} \int_{-\infty}^{t} e^{(1+\alpha) t^{\prime}} f\left(t^{\prime}\right) d t^{\prime}\right]
\end{aligned}
$$

with $\beta_{1}, \beta_{2} \in \mathbb{R}$ arbitrary. Now passing in the variable $x$ we obtain

$$
\begin{aligned}
u(x)= & x^{\alpha}\left[\beta_{1}+\frac{1}{2 \alpha-1} \int_{x}^{a} f\left(x^{\prime}\right)\left(x^{\prime}\right)^{1-\alpha} d x^{\prime}\right] \\
& +x^{1-\alpha}\left[\beta_{2}+\frac{1}{2 \alpha-1} \int_{0}^{x} f\left(x^{\prime}\right)\left(x^{\prime}\right)^{\alpha} d x^{\prime}\right] .
\end{aligned}
$$

The expression of $u$ can be written in the form

$$
u(x)=\beta_{1} x^{\alpha}+\frac{1}{2 \alpha-1} x^{\alpha} u_{1}(x)+\frac{1}{2 \alpha-1} x^{1-\alpha} u_{2}(x)+\beta_{2} x^{1-\alpha}
$$

with

and

$$
u_{1}(x)=\int_{x}^{a} f\left(x^{\prime}\right)\left(x^{\prime}\right)^{1-\alpha} d x^{\prime}
$$

From the hypothesis $b>\frac{3}{4}$ we deduce

$$
u_{2}(x)=\int_{0}^{x} f\left(x^{\prime}\right)\left(x^{\prime}\right)^{\alpha} d x^{\prime}
$$

$$
\alpha>\frac{3}{2}
$$

then the function $\beta_{1} x^{\alpha}$ is an element of $H^{2}(\Omega)$. Let us prove that $x^{\alpha} u_{1}, x^{1-\alpha} u_{2} \in L^{2}(\Omega)$. We are the following inequalities:

which gives

$$
\left|u_{1}(x)\right| \leq\left[\int_{x}^{a}\left(x^{\prime}\right)^{2-2 \alpha} d x^{\prime}\right]^{1 / 2}\|f\|_{L^{2}(\Omega)}
$$

$$
\left|u_{1}(x)\right| \leq \frac{x^{3 / 2-\alpha}}{\sqrt{2 \alpha-3}}\|f\|_{L^{2}(\Omega)}
$$


and we obtain in the same manner

$$
\left|u_{2}(x)\right| \leq \frac{x^{1 / 2+\alpha}}{\sqrt{2 \alpha+1}}\|f\|_{L^{2}(\Omega)} .
$$

We deduce from the above inequalities that $x^{\alpha} u_{1}, x^{1-\alpha} u_{2} \in L^{2}(\Omega)$ with

$$
\left\|x^{\alpha} u_{1}\right\|_{L^{2}(\Omega)}+\left\|x^{1-\alpha} u_{2}\right\|_{L^{2}(\Omega)} \leq \frac{a^{2}}{2}\left(\frac{1}{\sqrt{2 \alpha+1}}+\frac{1}{\sqrt{2 \alpha-3}}\right)\|f\|_{L^{2}(\Omega)} .
$$

Since $u$ must be an element of $L^{2}(\Omega)$ we necessarily have $\beta_{2}=0$, that is, $u$ is given by

$$
u(x)=\beta_{1} x^{\alpha}+\frac{1}{2 \alpha-1} x^{\alpha} u_{1}(x)+\frac{1}{2 \alpha-1} x^{1-\alpha} u_{2}(x) .
$$

We easily compute

$$
u^{\prime}(x)=\alpha \beta_{1} x^{\alpha-1}+\frac{\alpha}{2 \alpha-1} x^{\alpha-1} u_{1}(x)+\frac{1-\alpha}{2 \alpha-1} x^{-\alpha} u_{2}(x)
$$

and

(2.11) $u^{\prime \prime}(x)=-f(x)+\alpha(\alpha-1) \beta_{1} x^{\alpha-2}+\frac{\alpha(\alpha-1)}{2 \alpha-1}\left[x^{\alpha-2} u_{1}(x)+x^{-1-\alpha} u_{2}(x)\right]$.

With the help of (2.6) and (2.7) we obtain:

$\left|u^{\prime}(x)\right| \leq \alpha\left|\beta_{1}\right| x^{\alpha-1}+\left[\frac{\alpha}{2 \alpha-1} \frac{1}{\sqrt{2 \alpha-3}}+\frac{\alpha-1}{2 \alpha-1} \frac{1}{\sqrt{2 \alpha+1}}\right] x^{1 / 2}\|f\|_{L^{2}(\Omega)}, \quad \forall x \in \Omega$ and we deduce that $u^{\prime} \in L^{2}(\Omega)$ with

$$
\left\|u^{\prime}\right\|_{L^{2}(\Omega)} \leq\left|\beta_{1}\right| \frac{\alpha}{\sqrt{2 \alpha-1}} a^{\alpha-1 / 2}+\frac{a}{\sqrt{2}(2 \alpha-1)}\left(\frac{\alpha}{\sqrt{2 \alpha-3}}+\frac{\alpha-1}{\sqrt{2 \alpha+1}}\right)\|f\|_{L^{2}(\Omega)} .
$$

Now using the Hardy inequalities (see for exemple Lemma 6.2.1 of [7]), we infer that $x^{\alpha-2} u_{1}, x^{-1-\alpha} u_{2} \in L^{2}(\Omega)$ and

$$
\begin{aligned}
\left\|x^{\alpha-2} u_{1}\right\|_{L^{2}(\Omega)} & \leq \frac{2}{2 \alpha-3}\|f\|_{L^{2}(\Omega)} \\
\left\|x^{-1-\alpha} u_{2}\right\|_{L^{2}(\Omega)} & \leq \frac{2}{2 \alpha+1}\|f\|_{L^{2}(\Omega)} .
\end{aligned}
$$

Then from (2.11) we deduce that $u^{\prime \prime} \in L^{2}(\Omega)$ and

$$
\left\|u^{\prime \prime}\right\|_{L^{2}(\Omega)} \leq\left|\beta_{1}\right| \frac{\alpha(\alpha-1)}{\sqrt{2 \alpha-3}} a^{\alpha-3 / 2}+\left[1+\frac{4 \alpha(\alpha-1)}{(2 \alpha+1)(2 \alpha-3)}\right]\|f\|_{L^{2}(\Omega)}
$$

\section{The general dimensional case}

In this section we consider $n \in \mathbb{N}^{*}$ and we denote by $\Omega$ an open bounded domain included in $\mathbb{R}^{n+1}$, with boundary of class $C^{2}$.

Let us denote $\Gamma=\partial \Omega$; for any $x \in \Gamma$ we denote $\nu \equiv \nu(x) \in \mathbb{R}^{n+1}$ the normal vector in $x$ to $\Gamma$ oriented to the interior of $\Omega$. For any $\epsilon>0$ we denote

$$
\Sigma_{\epsilon}=\{x \in \Omega ; \operatorname{dist}(x, \Gamma)<\epsilon\} .
$$

It is well-known that there exists $\epsilon_{0}>0$ small enough such that for any $\left.\epsilon \in\right] 0, \epsilon_{0}[$ we have

$$
\Sigma_{\epsilon}=\{x+s \nu(x), x \in \Gamma, s \in] 0, \epsilon[\} .
$$


We also denote for any $\epsilon \in] 0, \epsilon_{0}[$

$$
\tilde{\Gamma}_{\epsilon}=\{x+\epsilon \nu(x), x \in \Gamma\} .
$$

Let us now give $a>0$ and consider $f \in L^{2}\left(\Sigma_{a}\right)$ and $u \in H^{1}\left(\Sigma_{a}\right)$ satisfying

$$
\left\{\begin{array}{ccc}
-\Delta u+b(x) u=f & \text { in } & H^{-1}\left(\Sigma_{a}\right) \\
u=0 & \text { for } & x \in \Gamma
\end{array}\right.
$$

In the above $b: \Sigma_{a} \rightarrow \mathbb{R}$ is a given regular enough function which "behaves as $\frac{1}{\operatorname{dist}^{2}(x, \Gamma)}$ for $x$ close" to $\Gamma$. For simplicity we suppose that there exists a function $b_{0}: \Gamma \rightarrow \mathbb{R}$ with $b_{0} \in C^{2}(\Gamma)$, such that

$$
\left.b(x+s \nu(x))=\frac{b_{0}(x)}{s^{2}}, \quad \forall x \in \Gamma, \forall s \in\right] 0, \min \left\{\epsilon_{0}, a\right\}[.
$$

In all this section we denote by $C$ a generic positive constant.

We have the following preliminary result:

LEMMA 3.1. Let us consider $f \in L^{2}\left(\Sigma_{a}\right)$ and $u \in H^{1}\left(\Sigma_{a}\right)$ satisfying (3.1).

a) For any $\left.a_{1} \in\right] 0, a[$ we have

$$
\|u\|_{H^{1}\left(\Sigma_{a_{1}}\right)} \leq C\left[\|u\|_{L^{2}\left(\Sigma_{a}\right)}+\|f\|_{L^{2}\left(\Sigma_{a}\right)}\right]
$$

b) For any $a_{1}, a_{2}$ with $0<a_{1}<a_{2}<a$, we have $u \in H^{2}\left(\Sigma_{1,2}\right)$ and

$$
\|u\|_{H^{2}\left(\Sigma_{1,2}\right)} \leq C\left[\|u\|_{L^{2}\left(\Sigma_{a}\right)}+\|f\|_{L^{2}\left(\Sigma_{a}\right)}\right]
$$

where we denoted $\Sigma_{1,2}=\Sigma_{a_{2}}-\overline{\Sigma_{a_{1}}}$.

Proof. a) We consider a cut-off function $\varphi \in C^{\infty}\left(\overline{\Sigma_{a}}\right)$ such that $\varphi \equiv 1$ on $\Sigma_{a_{1}}$ and $\varphi \equiv 0$ on $\partial\left(\Sigma_{a}\right)-\Gamma$. It is clear that $\tilde{u} \equiv u \varphi$ satisfies

$$
\left\{\begin{array}{ccc}
-\Delta \tilde{u}+b(x) \tilde{u}=\tilde{f} & \text { in } & \mathcal{D}^{\prime}\left(\Sigma_{a}\right) \\
\tilde{u}=0 & \text { for } & x \in \partial \Sigma_{a}
\end{array}\right.
$$

where $\tilde{f}=f \varphi-2 \nabla u \cdot \nabla \varphi-u \Delta \varphi$. We have that $\tilde{f}$ is an element of $H^{-1}\left(\Sigma_{a}\right)$ and

$$
\|\tilde{f}\|_{H^{-1}\left(\Sigma_{a}\right)} \leq c\left[\|u\|_{L^{2}\left(\Sigma_{a}\right)}+\|f\|_{L^{2}\left(\Sigma_{a}\right)}\right]
$$

From the Hardy inequality

$$
\left\|\frac{v}{\operatorname{dist}(x, \Gamma)}\right\|_{L^{2}\left(\Sigma_{a}\right)} \leq c\|v\|_{H^{1}\left(\Sigma_{a}\right)}, \quad \forall v \in H_{0}^{1}\left(\Sigma_{a}\right)
$$

we easily deduce that the problem (3.5) has an unique solution $\tilde{u} \in H_{0}^{1}\left(\Sigma_{a}\right)$ for any $\tilde{f} \in H^{-1}\left(\Sigma_{a}\right)$ and

$$
\|\tilde{u}\|_{H^{1}\left(\Sigma_{a}\right)} \leq c\|\tilde{f}\|_{H^{-1}\left(\Sigma_{a}\right)}
$$

With the help pf (3.6) we obtain the expected result.

b) This part is obvious by interior regularity.

We can now state the following result

TheOREM 3.2. Suppose that $b_{0}(x)>\frac{3}{4} \forall x \in \Gamma$. Then for any $f \in L^{2}\left(\Sigma_{a}\right)$ and $u \in H^{1}\left(\Sigma_{a}\right)$ satisfying (3.1) we have $u \in H^{2}\left(\Sigma_{a}\right)$ with

$$
\|u\|_{H^{2}\left(\Sigma_{a}\right)} \leq C\left[\|u\|_{L^{2}\left(\Sigma_{a}\right)}+\|f\|_{L^{2}\left(\Sigma_{a}\right)}\right]
$$


where $C \geq 0$ is a constant independent on $u$ and $f$.

We also have

$$
\frac{\partial u}{\partial \nu}=0 \quad \text { on } \Gamma
$$

Proof. From Lemma $3.1 \mathrm{~b})$ it suffices to prove that $u \in H^{2}\left(\Sigma_{s_{0}}\right)$ for $s_{0}>0$ small enough.

Let us consider the open sets $\Omega_{1}, \Omega_{2}, \cdots \Omega_{N}$ such that

$$
\Sigma_{s_{0}} \subset \Omega_{1} \cup \Omega_{2} \cdots \Omega_{N}
$$

and $\varphi_{1}, \varphi_{2}, \cdots \varphi_{N}$ a partition of unity associated to $\Omega_{1}, \Omega_{2}, \cdots \Omega_{N}$, with $\varphi_{k} \in \mathcal{D}\left(\Omega_{k}\right)$ and

$$
0 \leq \varphi_{k} \leq 1, \quad \sum_{k=1}^{N} \varphi_{k}=1 \text { on } \Sigma_{s_{0}} .
$$

Let us denote for any $k \in\{1,2, \cdots N\}$ :

$$
\tilde{\Omega}_{k}=\Omega_{k} \cap \Sigma_{s_{0}}, \quad \Gamma_{k}=\Omega_{k} \cap \Gamma
$$

and

$$
u_{k}=u \varphi_{k} .
$$

Since $u=\sum_{k=1}^{N} u_{k}$, to obtain the regularity result it suffices to prove that $u_{k} \in$ $H^{2}\left(\tilde{\Omega}_{k}\right)$ and to obtain appropriate estimates for $\left\|u_{k}\right\|_{H^{2}\left(\tilde{\Omega}_{k}\right)}$.

It is clear that

$$
\left\{\begin{array}{ccc}
-\Delta u_{k}+b u_{k}=f_{k} & \text { in } & \tilde{\Omega}_{k} \\
u_{k}=0 & \text { on } & x \in \partial \tilde{\Omega}_{k}-\tilde{\Gamma}_{s_{0}}
\end{array}\right.
$$

where we denoted

$$
f_{k}=f \varphi-2 \nabla u \cdot \nabla \varphi_{k}-u \Delta \varphi_{k}
$$

We can suppose that

$$
\tilde{\Omega}_{k}=\left\{x+s \nu(x), \quad x \in \Gamma_{k}, \quad s \in[0, a]\right\} .
$$

We also suppose that every set $\Gamma_{k}$ is an $n$-dimensional $C^{2}$ - manifold which can be written in the following manner:

$$
\Gamma_{k}=\left\{g^{k}(t), \quad t \in T_{k}\right\}
$$

where $T_{k}$ is a bounded regular open set in $\mathbb{R}^{n}$ and $g^{k}: T_{k} \rightarrow \mathbb{R}^{n+1}$ is an injective and $C^{2}$ - function (for exemple $\Gamma_{k}$ can be defined as a $C^{2}$ - graph in an appropriate local coordinates system).

We now introduce the vectors in $\mathbb{R}^{n+1}$ :

$$
\tau_{j} \equiv \tau_{j}(t)=\frac{\partial g^{k}}{\partial t_{j}}(t), \quad j=1, \cdots n
$$

These vectors are tangent to the manifold $\Gamma_{k}$ and we suppose that $\tau_{1}(t), \cdots \tau_{n}(t)$ are independent in $\mathbb{R}^{n+1}$ for any $t \in T_{k}$. Let us define

$$
\tilde{\nu} \equiv \tilde{\nu}(t)=\tau_{1} \wedge \tau_{2} \cdots \wedge \tau_{n}
$$

(the vectorial product in $\mathbb{R}^{n+1}$ ) where we recall that $\tilde{\nu}_{j}=(-1)^{k+1} \operatorname{det}\left(\mathbf{M}_{j}\right)$ with $\mathbf{M}_{j}$ the $n \times n$ matrix obtained from $\mathbf{M}$ by suppresing the line $j$, where $\mathbf{M}$ is the 
$(n+1) \times n$ matrix whose $l$-column is $\tau_{l}$.

It is clear that $\tilde{\nu}$ is normal to $\Gamma_{k}$ and we suppose that $\nu$ is given by

$$
\nu=(-1)^{n} \frac{\tilde{\nu}}{d}
$$

where we denote $d \equiv d(t)=\|\tilde{\nu}(t)\|>0 \quad($ since $\tilde{\nu}(t) \neq 0)$.

Let us denote $Q=T_{k} \times\left[0, s_{0}\right]$. We suppose that $s_{0}>0$ is small enough such that the function $\theta: Q \rightarrow \tilde{\Omega}_{k}$ given by

$$
\theta(t, s)=g(t)+s \nu(t)
$$

is injective. It is easy to see that the Jacobian matrix of $\theta$ is the matrix $\mathbf{A} \equiv \mathbf{A}(t, s)$ given by

$$
\mathbf{A}(t, s)=\mathbf{A}_{0}(t)+s \mathbf{J}(t)
$$

where $\mathbf{A}_{0}$ and $\mathbf{J}_{0}$ are written by columns as

$$
\mathbf{A}_{0} \equiv \mathbf{A}_{0}(t)=\left(\tau_{1}(t) \cdots \tau_{n}(t) \nu(t)\right)
$$

and

We also have

$$
\mathbf{J}_{0} \equiv \mathbf{J}_{0}(t)=\left(\frac{\partial \nu}{\partial t_{1}}(t) \cdots \frac{\partial \nu}{\partial t_{n}}(t) 0\right)
$$

$$
\operatorname{det}\left(\mathbf{A}_{0}\right)=\sum_{j=1}^{n+1}(-1)^{n+j+1} \nu_{j} \operatorname{det}\left(\mathbf{M}_{j}\right)=\sum_{j=1}^{n+1}(-1)^{n} \nu_{j} \tilde{\nu}_{j}=d(t)>0, \quad \forall t \in T_{k} .
$$

By continuity we deduce that there exists $d_{0}>0$ such that

$$
\operatorname{det}(\mathbf{A}(t, s)) \geq d_{0} \quad \text { on } \quad Q
$$

for $s_{0}$ small enough. Then the function $\theta$ is a diffeomorphisme between $Q$ and $\tilde{\Omega}_{k}$.

Let us denote $\mathbf{B} \equiv \mathbf{B}(t, s)=\mathbf{A}^{-1}$. It is well-known that the laplacian operator on $\tilde{\Omega}_{k}$ is given in coordinates $(t, s)$ by

$$
\Delta_{x} v=L(v)
$$

where we set

$$
L(v)=\frac{1}{\operatorname{det} \mathbf{A}} \nabla_{t, s} \cdot\left[(\operatorname{det} \mathbf{A}) \mathbf{B B}^{T} \nabla_{t, s} v\right]
$$

Then the equation (3.10) is written in coordinates $(t, s)$ under the form

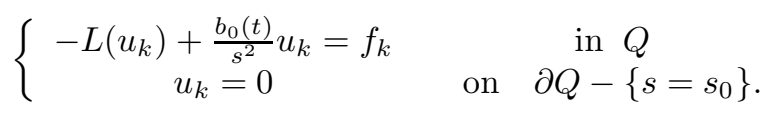

It is clear that for $s_{0}$ small enough, we have for any $m \in \mathrm{N}$ that $v \in H^{m}\left(\tilde{\Omega}_{k}\right) \Longleftrightarrow v \in H^{m}(Q)$ with equivalence of norms.

Let us observe that

$$
\mathbf{A}_{0}^{T} \mathbf{A}_{0}=\left(\begin{array}{cc}
\mathbf{R}(t) & 0 \\
0 & 1
\end{array}\right)
$$

where $\mathbf{R} \equiv \mathbf{R}(t)$ is the $n \times n$ invertible matrix given by

$$
\mathbf{R}_{i j}(t)=\tau_{i}(t) \cdot \tau_{j}(t), \quad i, j=1, \cdots n .
$$

Since $\mathbf{B B}^{T}=\left(\mathbf{A}^{T} \mathbf{A}\right)^{-1}$ we deduce

$$
\mathbf{B B}^{T}=\left(\mathbf{A}_{0}^{T} \mathbf{A}_{0}\right)^{-1}+s O(1)
$$


where we denote in all this paper by $\mathrm{O}(1)$ terms which are regular enough on $\overline{\Sigma_{s_{0}}}$. We remark that

$$
\operatorname{det} \mathbf{A}=d(t)+s O(1) .
$$

By a direct calculus we can prove that the operator $L$ can be written in the form

$$
L(v)=\frac{\partial^{2} v}{\partial s^{2}}+L_{0}(v)+L_{1}(v)+s L_{2}(v)
$$

where

$$
L_{0}(v)=\frac{1}{d} \nabla_{t} \cdot\left(d \mathbf{R} \nabla_{t} v\right)
$$

$L_{1}$ is a first order linear differential operator in $(t, s)$

$L_{2}$ is a second order linear differential operator in $(t, s)$.

The goal is now to prove that $f_{k}, L_{0}\left(u_{k}\right), L_{1}\left(u_{k}\right)$ and $s L_{2}\left(u_{k}\right)$ are in $L^{2}(Q)$, to obtain appropiate $L^{2}$ - estimates for these expressions and to conclude using the results of Theorem 2.1.

From Lemma 3.1 we deduce that

$$
\left\|u_{k}\right\|_{H^{1}(Q)} \leq C\left[\|u\|_{L^{2}\left(\Sigma_{a}\right)}+\|f\|_{L^{2}\left(\Sigma_{a}\right)}\right]
$$

and

$$
\left\|u_{k}\right\|_{H^{3 / 2}\left(\left\{s=s_{0}\right\}\right)} \leq C\left[\|u\|_{L^{2}\left(\Sigma_{a}\right)}+\|f\|_{L^{2}\left(\Sigma_{a}\right)}\right]
$$

which allows to obtain $f_{k}, L_{1}\left(u_{k}\right) \in L^{2}(Q)$ with

$$
\left\|f_{k}\right\|_{L^{2}(Q)}+\left\|L_{1}\left(u_{k}\right)\right\|_{L^{2}(Q)} \leq C\left[\|u\|_{L^{2}\left(\Sigma_{a}\right)}+\|f\|_{L^{2}\left(\Sigma_{a}\right)} \cdot\right]
$$

Let us now observe that for any $v$ we have

$$
L(s v)=s L(v)+L_{3}(v)
$$

and

$$
L_{2}(s v)=s L_{2}(v)+L_{4}(v)
$$

with $L_{3}, L_{4}$ first order linear differential operators in $(t, s)$. Then multiplying (3.12) by $s$ and using (3.18) we obtain

$$
\left\{\begin{array}{cc}
-L\left(s u_{k}\right)=s f_{k}-\frac{b_{0}(t)}{s} u_{k}-L_{3}\left(u_{k}\right) & \text { in } Q \\
s u_{k}=0 & \text { on } \quad \partial Q-\left\{s=s_{0}\right\} .
\end{array}\right.
$$

Now we have the following Hardy inequality

$$
\left\|\frac{v}{s}\right\|_{L^{2}(Q)} \leq C\|v\|_{H^{1}(Q)} \quad \forall v \in H^{1}(Q) \text { with } v=0 \text { on }\{s=0\}
$$

which implies

$$
\left\|\frac{v}{s^{2}}\right\|_{H^{-1}(Q)} \leq C\|v\|_{H^{1}(Q)} \quad \forall v \in H^{1}(Q) \text { with } v=0 \text { on }\{s=0\} .
$$

From (3.15), (3.16) and (3.21) we deduce by classical regularity that $s u_{k} \in H^{2}(Q)$. Then using (3.19) we deduce that $s L_{2}\left(u_{k}\right) \in L^{2}(Q)$ and

$$
\left\|s L_{2}\left(u_{k}\right)\right\|_{L^{2}(Q)} \leq C\left[\|u\|_{L^{2}\left(\Sigma_{a}\right)}+\|f\|_{L^{2}\left(\Sigma_{a}\right)}\right] .
$$

On the other hand, we remark that for any $j=1, \cdots n$ we have

$$
\frac{\partial}{\partial t_{j}} L(v)=L\left(\frac{\partial v}{\partial t_{j}}\right)+L_{5}(v)
$$


with $L_{5}$ a second order linear differential operators in $(t, s)$. Now deriving (3.12) with respect to $t_{j}$ we deduce

$$
\left\{\begin{array}{cc}
-L\left(\frac{\partial u_{k}}{\partial t_{j}}\right)+\frac{b_{0}(t)}{s^{2}} \frac{\partial u_{k}}{\partial t_{j}}=\frac{\partial f_{k}}{\partial t_{j}}+L_{5}\left(u_{k}\right)-\frac{u_{k}}{s^{2}} \frac{\partial b_{0}}{\partial t_{j}} & \text { in } Q \\
\frac{\partial u_{k}}{\partial t_{j}}=0 & \text { on } \quad \partial Q-\left\{s=s_{0}\right\} .
\end{array}\right.
$$

Denoting by $g_{k}$ the right-hand part of the first equation of (3.24) we prove, with the help of $(3.22)$, that $g_{k} \in H^{-1}(Q)$ and

$$
\left\|g_{k}\right\|_{H^{-1}(Q)} \leq C\left[\|u\|_{L^{2}\left(\Sigma_{a}\right)}+\|f\|_{L^{2}\left(\Sigma_{a}\right)}\right] .
$$

We now use the fact that the operator $-L v+\frac{b_{0}}{s^{2}} v$ is an isomorphisme from $H_{0}^{1}(Q)$ to $H^{-1}(Q)$ and that $\frac{\partial u_{k}}{\partial t_{j}} \in H^{1 / 2}\left(\left\{s=s_{0}\right\}\right)$ with

$$
\left\|\frac{\partial u_{k}}{\partial t_{j}}\right\|_{H^{1 / 2}\left(\left\{s=s_{0}\right\}\right)} \leq C\left[\|u\|_{L^{2}\left(\Sigma_{a}\right)}+\|f\|_{L^{2}\left(\Sigma_{a}\right)}\right]
$$

as a consequence of Lemma 3.1. Then for any $j=1, \cdots n$ we deduce that $\frac{\partial u_{k}}{\partial t_{j}} \in$ $H^{1}(Q)$, and we obtain $L_{0}\left(u_{k}\right) \in L^{2}(Q)$ with

$$
\left\|L_{0}\left(u_{k}\right)\right\|_{L^{2}(Q)} \leq C\left[\|u\|_{L^{2}\left(\Sigma_{a}\right)}+\|f\|_{L^{2}\left(\Sigma_{a}\right)}\right] .
$$

Now the equation (3.12) can be written in the form

$$
\left\{\begin{array}{c}
-\frac{\partial^{2} u_{k}}{\partial s^{2}}+\frac{b_{0}(t)}{s^{2}} u_{k}=h_{k} \quad \text { in } Q \\
u_{k}(s=0)=0
\end{array}\right.
$$

with

$$
h_{k}=f_{k}+L_{1}\left(u_{k}\right)+s L_{2}\left(u_{k}\right)+L_{0}\left(u_{k}\right) \text {. }
$$

From (3.17), (3.23) and (3.25) we deduce that $h_{k} \in L^{2}(Q)$ and

$$
\left\|h_{k}\right\|_{L^{2}(Q)} \leq C\left[\|u\|_{L^{2}\left(\Sigma_{a}\right)}+\|f\|_{L^{2}\left(\Sigma_{a}\right)}\right]
$$

which allows to write $h_{k}(t, \cdot) \in L^{2}(] 0, s_{0}[)$ a.e. $t \in T$.

Since $u_{k}(t, \cdot) \in L^{2}(] 0, s_{0}[)$ we can apply Theorem 2.1 and deduce that $u_{k}(t, \cdot) \in$ $H^{2}(] 0, s_{0}[)$ a.e. $t \in T$. We also have a.e. $t \in T_{k}$ :

$$
\left\|\frac{\partial^{2} u_{k}(t, \cdot)}{\partial s^{2}}\right\|_{L^{2}(] 0, s_{0}[)} \leq C_{1}\left(s_{0}, \alpha\right)\left\|h_{k}(t, \cdot)\right\|_{L^{2}(] 0, s_{0}[)}+C_{2}\left(s_{0}, \alpha\right)\left\|u_{k}(t, \cdot)\right\|_{L^{2}(] 0, s_{0}[)}
$$

with $\alpha \equiv \alpha(t)=\frac{1}{2}\left(1+\sqrt{b_{0}(t)}\right)$ and $C_{1}, C_{2}$ given in Theorem 2.1. We also obtain $\frac{\partial u_{k}}{\partial s}(t, 0)=0$ a.e. $t \in T_{k}$ which gives (3.9), where we use the fact that $\mathbf{B}(t, 0) \nu=$ $(0, \cdots, 0,1)^{T}$. Integrating in $T_{k}$ and using $(3.27)$ we easily obtain that $u$ belongs to $H^{2}\left(\Sigma_{a}\right)$ and satisfies (3.8).

\section{An application to a FENE model for diluted polymers}

We consider the stationary Fokker-Planck-Smoluchowski equation in $\mathbb{R}^{d}, d=2$ or $d=3$, which comes from the modelisation of the diluted polymers where the molecules are considered as elastic springs (FENE models, see for exemple [3], [4], [5] and [6]). We suppose that

(1) The length of the molecules are no larger than a physical constant supposed equal to 1 by normalization. 
(2) The gradient of the velocity of the fluid is a constant traceless $d \times d$ matrix denoted by $\mathbf{G}$.

(3) The force in the elastic springs is given by

$$
F(x)=\frac{2 \delta x}{1-\|x\|^{2}}, \quad \forall x \in B
$$

where we denote $\delta>0$ a physical constant and $B=B(0,1)$ the ball in $\mathbb{R}^{d}$ centered in 0 with radius 1 .

We search for a density probability $\psi$ defined on $B$ solution of

$$
\left\{\begin{array}{clc}
-\Delta \psi-\nabla \cdot[F(x) \psi-\mathbf{G} x \psi]=0 & \text { for } & x \in B \\
\frac{\partial \psi}{\partial x}+[F(x) \psi-\mathbf{G} x \psi] \cdot x=0 & \text { for } & x \in \partial B
\end{array}\right.
$$

Remark that $F(x)=\nabla \phi(x)$ with $\phi(x)=-\delta \log \left(1-\|x\|^{2}\right)$, then the problem (4.1) can be written in the form

$$
\left\{\begin{array}{cll}
-\nabla \cdot\left[M \nabla\left(\frac{\psi}{M}\right)\right]+\nabla \cdot(\mathbf{G} x \psi)=0 & \text { for } & x \in B \\
{\left[-M \nabla\left(\frac{\psi}{M}\right)+\mathbf{G} x \psi\right] \cdot x=0} & \text { for } & x \in \partial B
\end{array}\right.
$$

where we denote $M(x)=\left(1-\|x\|^{2}\right)^{\delta}$. This equation has to be completed by the conditions:

$$
\begin{gathered}
\psi \geq 0 \\
\int_{B} \psi(x) d x=q
\end{gathered}
$$

with $q>0$ a given constant.

Let us now introduce the following functional spaces

$$
\begin{gathered}
L_{M}^{2} \equiv L_{M}^{2}(B):=\left\{\varphi \in L_{\mathrm{loc}}^{1}(B), \int_{B} \frac{\varphi^{2}}{M} d x<\infty\right\} \\
H_{M}^{1} \equiv H_{M}^{1}(B):=\left\{\varphi \in L_{\mathrm{loc}}^{1}(B), \int_{B}\left[\frac{\varphi^{2}}{M}+M\left|\nabla\left(\frac{\varphi}{M}\right)\right|^{2}\right] d x<\infty\right\} .
\end{gathered}
$$

Then the variational formulation of the problem (4.2) is: find $\psi \in H_{M}^{1}$ such that

$$
\int_{B}\left[M \nabla\left(\frac{\psi}{M}\right) \cdot \nabla\left(\frac{\varphi}{M}\right)-\mathbf{G} x \psi \cdot \nabla\left(\frac{\varphi}{M}\right)\right] d x=0 \quad \forall \varphi \in H_{M}^{1} .
$$

The existence and uniqueness of a solution of (4.5) satisfying also (4.3) and (4.4) was given in [2] and [3].

The goal of this section is to give a supplementary regularity result for $\psi$. Let us begin by the following preliminary result:

Lemma 4.1. For any $\delta>1$ we have

$$
H_{M}^{1}=\left\{\varphi \in L_{l o c}^{1}(B), \frac{\varphi}{\sqrt{M}} \in H_{0}^{1}(B)\right\}
$$

and there exist constants $0<c_{1}<c_{2}$ such that

$$
c_{1}\left\|\frac{\varphi}{\sqrt{M}}\right\|_{H_{1}(B)} \leq\|\varphi\|_{H_{M}^{1}} \leq c_{2}\left\|\frac{\varphi}{\sqrt{M}}\right\|_{H_{1}(B)}, \forall \varphi \in H_{M}^{1} .
$$


Proof. Let us consider $\varphi \in H_{M}^{1}$ arbitrary. We have

$$
\nabla\left(\frac{\varphi}{\sqrt{M}}\right)=\sqrt{M} \nabla\left(\frac{\varphi}{M}\right)-\frac{\nabla M}{2 M^{3 / 2}} \varphi
$$

Since $\nabla M=-2 \delta x M^{1-1 / \delta}$ we obtain

$$
\left\|\frac{\varphi}{\sqrt{M}}\right\|_{H^{1}(B)}^{2} \leq \int_{B} \frac{\varphi^{2}}{M}+2 \int_{B} M\left|\nabla\left(\frac{\varphi}{M}\right)\right|^{2}+2 \delta^{2} \int_{B} \frac{\|x\|^{2}}{M^{1+2 / \delta}} \varphi^{2}
$$

Now using Theorem 6.2.5 of [7] (see also the inclusion (3.10) of [3]) we deduce that $\frac{\varphi}{\sqrt{M}} \in H^{1}(B)$ and that the first inequality of (4.6) is satisfied.

On the other hand, from the density of $\mathcal{D}(B)$ in $H_{M}^{1}$ (see Remark 3.7 of [6]) we deduce that there exists a sequence $\varphi_{k} \in \mathcal{D}(B)$ such that $\varphi_{k} \rightarrow \varphi$ in $H_{M}^{1}$. Then

$$
\frac{\varphi_{k}}{\sqrt{M}} \rightarrow \frac{\varphi}{\sqrt{M}} \quad \text { in } H^{1}(B) \quad \text { with } \frac{\varphi_{k}}{\sqrt{M}} \in \mathcal{D}(B)
$$

and this implies $\frac{\varphi}{\sqrt{M}} \in H_{0}^{1}(B)$.

Let us now consider $v \in H_{0}^{1}(B)$ and denote $\varphi=\sqrt{M} v$. We have

$$
\int_{B} \frac{\varphi^{2}}{M}+\int_{B} M\left|\nabla\left(\frac{\varphi}{M}\right)\right|^{2}=\int_{B} v^{2}+\int_{B}|\nabla v|^{2}+\frac{1}{4} \int_{B}\left|\frac{\nabla M}{M}\right|^{2} v^{2} .
$$

With the help of the Hardy inequality we deduce that $\varphi \in H_{M}^{1}$ and obtain the second inequality of (4.6).

Then using the changes $\psi=\sqrt{M} f$ and $\varphi=\sqrt{M} g$, the problem (4.5) can be written in the equivalent form: find $f \in H_{0}^{1}(B)$ such that

$$
\int_{B}\left[M \nabla\left(\frac{f}{\sqrt{M}}\right) \cdot \nabla\left(\frac{g}{\sqrt{M}}\right)-\mathbf{G} x \sqrt{M} f \cdot \nabla\left(\frac{g}{\sqrt{M}}\right)\right] d x=0 \quad \forall g \in H_{0}^{1}(B) .
$$

By an elementary calculus, the above equality writes

$\int_{B}\left\{\nabla f \cdot \nabla g-\mathbf{G} x f \cdot \nabla g+\left[\left(\delta^{2}-2 \delta\right) M^{-2 / \delta}-\left(\delta^{2}+(n-2) \delta+\delta \mathbf{G} x \cdot x\right) M^{-1 / \delta}\right] f g\right\}$ then $f \in H_{0}^{1}(B)$ satisfies the problem

$$
-\Delta f+\mathbf{G} x \cdot \nabla f+\left[\frac{\delta^{2}-2 \delta}{\left(1-\|x\|^{2}\right)^{2}}-\frac{\delta^{2}+(n-2) \delta+\delta \mathbf{G} x \cdot x}{1-\|x\|^{2}}\right] f=0 \quad \text { in } H^{-1}(B) .
$$

Let us now write

$$
\frac{1}{\left(1-\|x\|^{2}\right)^{2}}=\frac{1}{(1+\|x\|)^{2}} \frac{1}{(1-\|x\|)^{2}}=\frac{1}{4} \frac{1}{(1-\|x\|)^{2}}+\frac{3+\|x\|}{4(1+\|x\|)^{2}} \frac{1}{1-\|x\|}
$$

and observe that $\operatorname{dist}(x, \partial B)=1-\|x\|, \quad \forall x \in B$. Since $\frac{f}{1-\|x\|} \in L^{2}(B)$ by Hardy inequality, we deduce that $f$ satisfies

$$
-\Delta f+\frac{\delta^{2}-2 \delta}{4 \operatorname{dist}^{2}(x, \partial B)} f=h \quad \text { in } H^{-1}(B)
$$

with $h \in L^{2}(B)$.

Then the result of Theorem 3.2 applies with $b_{0}=\frac{\delta^{2}-2 \delta}{4}$ provided that $\frac{\delta^{2}-2 \delta}{4}>\frac{3}{4} \Longleftrightarrow \delta>3$ and we get $f \in H^{2}(B)$ and $\frac{\partial f}{\partial \nu}=0$ on $\partial B$.

We then proved the following regularity result: 
Proposition 4.2. Under the hypothesis $\delta>3$ the solution $\psi$ of (4.5) satisfies

$$
\frac{\psi}{\sqrt{M}} \in H_{0}^{2}(B)
$$

\section{Acknowledgements}

The author thanks to Petru Mironescu for useful talks on regularity results in elliptic PDEs.

\section{References}

[1] L. Boccardo, L. Orsina, I. Peral, A remark on existence and optimal summability of solutions of elliptic problems involving Hardy potential, Discrete and continuous Dynamical Systems, Vol. 16, No. 3, (2006), 513-523.

[2] L. Chupin, The FENE model for viscoelastic thin film flows, Methods Appl. Anl. 16 (2009) no. 2, 217-261.

[3] I.S. Ciuperca and L.I. Palade, The steady state configurational distribution diffusion equation of the standard FENE dumbbell polymer model: existence and uniqueness of solutions for arbitrary velocity gradients, Math. Mod. Meth. Appl. Sci. (M3AS), Vol. 19, No. 11 (2009) 2039-2064.

[4] B. Jourdain, C. Le Bris, T. Lelièvre, F. Otto, Long-Time asymptotics of a multiscale model for a polymeric fluid flows, Arc. Rational Mech. Anal. 181 (2006) 97-148.

[5] F. Lin, C. Liu, P. Zhang, On a micro-macro model for polymeric fluids near equilibrium, Comm. Pure Appl. Math. 60 (2007), no. 6, 838866.

[6] N. Masmoudi, Well-Posedness for the FENE dumbbell model of polymeric flows, Comm. Pure Appl. Math. 61 (12) (2008), 1685-1714.

[7] J. Nečas, Les méthodes diréctes en théorie des équations elliptiques, Masson, Paris, 1967.

Institut Camille Jordan, Université de Lyon, France

E-mail address: ciuperca@math.univ-lyon1.fr 